\title{
Shared Decision Making
}

\section{Ausganglage}

Shared Decision Making (SDM) ist ein Modell der Entscheidungsfindung im klinischen Kontext, gemäss welchem Arzt ${ }^{1}$ und Patient - wenn möglich gemeinsam mit dessen Angehörigen - aktiv Informationen austauschen, verschiedene Behandlungsoptionen abwägen und partnerschaftlich eine Entscheidung fällen. Der Patient bringt sein subjektives Wissen zu seiner Erkrankung, seine Werte und Bedürfnisse in die Diskussion ein. Der Arzt vermittelt fachliches Wissen, klinische Erfahrung und trägt durch eine objektive Distanz zur klinischen Problematik und zum Patienten zu einer optimalen Entscheidungsfindung bei. Dennoch besteht häufig eine gewisse Informationsasymmetrie zwischen Arzt und Patient welche je nach Patientengruppe unterschiedlich stark ausgeprägt sein kann. Deshalb ist es entscheidend für das Gelingen von SDM, dass der Arzt während der ganzen Konsultation eine Atmosphäre schafft, in welcher sich der Patient frei äussern kann.

SDM bietet den Vorteil, dass die Patienten zwar eine aktive Rolle haben, sie aber in der Entscheidungsfindung nicht allein gelassen werden. Aus ethischen Gründen ist SDM deshalb sehr sinnvoll und entspricht einem zunehmenden Bedürfnis von Patienten. SDM ist aber kein Wundermittel. Wenn sogenannte Entscheidungshilfen eingesetzt werden, erhöht SDM das Wissen der Patienten über die Erkrankung, fördert deren aktiven Einbezug und hilft ihnen, Präferenzen zu reflektieren. Es gibt Hinweise, dass durch SDM unnötige Behandlungen verhindern werden können. SDM hat aber gemäss aktueller
Evidenz weder klar positive noch negative Auswirkungen auf Gesundheitszustand, Wohlbefinden und Zufriedenheit der Patienten.

\section{Position der FMH}

SDM ist eine patientenzentrierte Methode, welche sich auf Entscheidungssituationen bezieht. Die FMH erachtet weitere patientenzentrierte Methoden und Konzepte wie Gesundheitskompetenz, Patientenempowerment oder Motivational Interviewing ebenfalls als wichtig, da sie sich gegenseitig ergänzen und optimalerweise miteinander kombiniert werden.

SDM beschreibt ein Idealmodell. Für den ärztlichen Alltag sind folgende Aspekte aus Sicht der FMH hervorzuheben:

\section{Patientenpräferenzen miteinbeziehen}

Wenn eine Entscheidung zwischen mindestens zwei vergleichbaren Behandlungsoptionen getroffen werden muss, werden die verschiedenen Vor- und Nachteile gegeneinander abgewogen. Patienten gewichten diese in Abhängigkeit von ihren Werten, Präferenzen und Bedürfnissen unterschiedlich. SDM ist hier ein sinnvolles Vorgehen, um eine solche präferenzsensitive Entscheidung zu treffen und die von den Patienten gewünschten Behandlungsziele zu erreichen.

Damit Arzt und Patient gemäss dem SDM-Modell gemeinsam eine Behandlungsentscheidung treffen können, muss genügend Spielraum für verschiedene Behandlungsoptionen vorhanden sein. Initiativen zur Verbesserung der Qualität sollten deshalb
1 Zur besseren Lesbarkeit wird in der Regel die männliche Form verwendet; Frauen sind immer mitgemeint
Korrespondenz:

FMH/DDQ

Elfenstrasse 18

CH-3000 Bern 15

Tel. 0313591111

Fax 0313591112

ddq[at]fmh.ch

www.fmh.ch
Neben SDM gibt es weitere wichtige patientenzentrierte Konzepte und Methoden wie Gesundheitskompetenz, Patientenempowerment oder Motivational Interviewing, die sich gegenseitig ergänzen.

- SDM ist ein Idealmodell. Es ist grundsätzlich ein sinnvolles Vorgehen, um präferenzsensitive Entscheidungen zu treffen und die von den Patienten gewünschten Behandlungsziele zu erreichen. Patienten und wenn möglich auch die Angehörigen werden aktiv miteinbezogen, ohne sie im Entscheidungsfindungsprozess allein zu lassen.

- Ärztinnen und Ärzte sollen Patienten ermutigen, sich in Entscheidungssituationen aktiv einzubringen. Wer im Einzelfall die Behand- lungsentscheidung trifft, sollte aber laufend der klinischen Situation und den Bedürfnissen der Patienten angepasst werden.

- Patientenpräferenzen sollten bei der Entwicklung von Guidelines explizit miteinbezogen werden.

- Ärztinnen und Ärzte tragen für die sorgfältige Behandlung ihrer Patienten die Verantwortung. Damit sie gemeinsam mit den Patienten auf deren Präferenzen abgestimmte Behandlungsentscheidungen treffen können, müssen sie ihre Patienten, unter Berücksichtigung der bestehenden Informationsasymmetrie, nachweisbar korrekt aufklären (Aufklärungspflicht). 


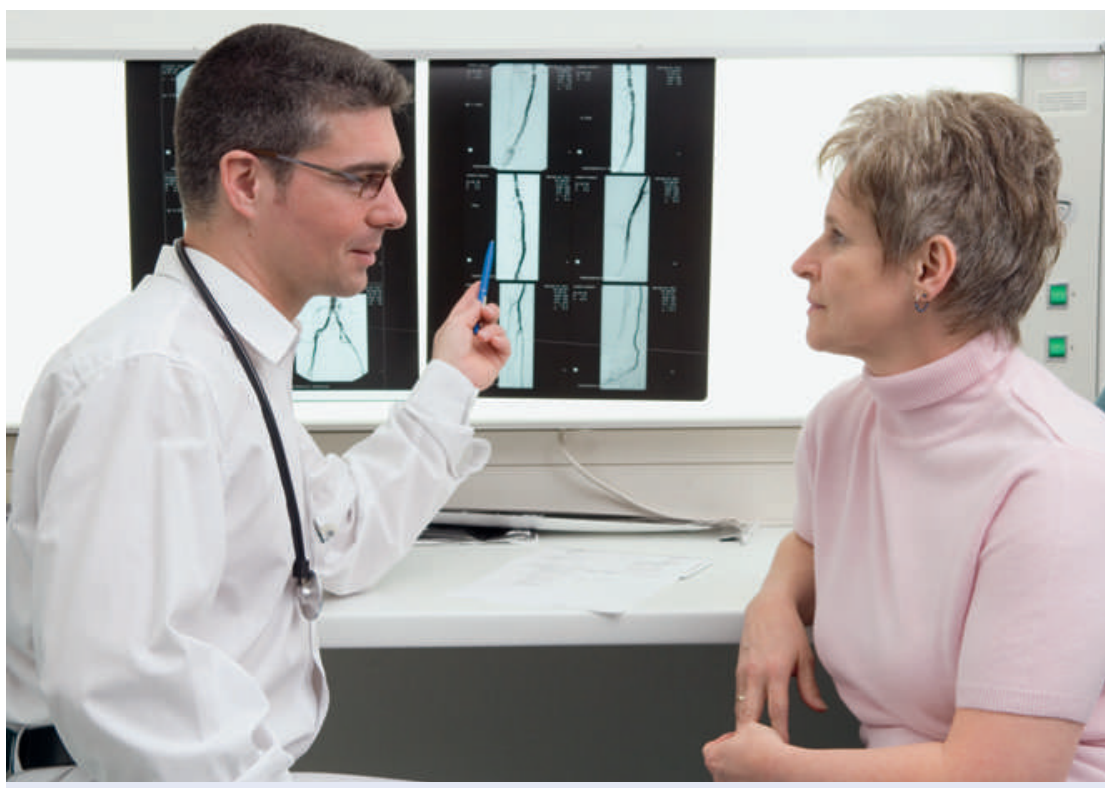

Shared Decicion Making: Informationen austauschen, verschiedene Behandlungsoptionen abwägen, partnerschaftlich eine Entscheidung fällen. es Situationen, in welchen ein Teil der Patienten die Entscheidung lieber dem Arzt überlassen möchte. Der Einbezug der Patienten in Entscheidungen sollte deshalb stets an deren Wünsche angepasst werden. Die Anwendung der Modelle zur Entscheidungsfindung sollte deshalb flexibel gehandhabt werden und laufend die Bedürfnisse der Patienten berücksichtigen.

Fehlende wissenschaftliche Studien und Ressourcen Es gibt zwar sehr viele wissenschaftliche Studien zur Wirksamkeit von Entscheidungshilfen, doch für den praktischen Einsatz in vielen Bereichen fehlen Entscheidungshilfen von hoher Qualität. Obwohl diese für SDM nicht zwingend nötig sind, erleichtern sie die Vermittlung von verständlichen und umfassenden Informationen. Die Entwicklung und Verbreitung von Entscheidungshilfen für Patienten sollte deshalb gefördert werden.

Im Gegensatz dazu gibt es wenig spezifische Forschung zu SDM. Für die Umsetzung von SDM wäre es hilfreich, wenn die Forschung differenzierte Hinweise zur Wirksamkeit von SDM, zum Beispiel für bestimmte Patientengruppen, liefern könnte. Insbesondere fehlen auch wissenschaftlich basierte Antworten nach einer optimalen Art, um SDM zu implementieren und effektive Trainingsprogramme zu gestalten.

\section{Verantwortung und Haftung}

Ärztinnen und Ärzte tragen für die sorgfältige Behandlung ihrer Patienten die Verantwortung. Sie sollten gemeinsam mit den Patienten auf deren Präferenzen abgestimmte Behandlungsentscheidungen treffen können, ohne mögliche Haftpflichtansprüche oder Schadenersatzforderungen fürchten $\mathrm{zu}$ müssen, etwa wenn Patienten bei einer Entscheidung die Lebensqualität höher gewichten als die Lebensdauer. Deshalb ist es wichtig, dass Ärzte ihre Patienten korrekt aufklären (Aufklärungspflicht, BGE $117 \mathrm{Ib}$ 197) und dies bei Bedarf nachweisen können. 